811.133.1'366.596:811.163.41'367.625 https://doi.org/10.18485/sj.2018.23.1.24

MILANA L. DODIG*

Université de Kragujevac

Faculté des Lettres et des Arts
Оригинални научни рад

Примљен: 04. 01. 2018.

Прихваћен: 29. 01. 2018.

\title{
ASPECT DANS LES SYSTÈMES SERBE ET FRANÇAIS ET SON RÔLE DANS LE CAS DU CONDITIONNEL ET DU POTENITEL
}

Dans ce travail nous examinons la question de l'aspect dans les langues français et serbe. En expliquant les différences concernant cette catégorie, nous essaierons de démontrer également le fonctionnement aspectuel du conditionnel français et du potentiel serbe.

Mots clés : aspect grammatical / lexical, conditionnel français, potentiel serbe.

\section{INTRODUCTION}

Il est notoire que dans la description d'une forme verbale participent les différentes catégories, à savoir les catégories de la personne, du nombre, du mode, du temps, de l'aspect et de la voix (Riegel 1994 : 287). Le temps, le mode et l'aspect représentent des catégories étroitement liées, même si dans la littérature traditionnelle celles-ci ont été traitées de façon indépendante, et exigent un traitement unitaire pour pouvoir procéder à la représentation d'une forme verbale. Nous allons ici attribuer de l'importance à la question de l'aspect.

*dodigmilana@yahoo.com 
Il est à noter que la notion d'aspect représente un sujet problématique dans la littérature française étant donné les nombreuses divergences d'analyse et de terminologie parmi les linguistes. À notre avis, toutes les divergences relèvent de l'approche sémasiologique appliquée traditionnellement, même aujourd'hui. Rares sont les études typologiques ou onomasiologiques consacrées à l'aspect. Pour cette raison, nous soutenons l'approche de Tournadre (2004) traitant de l'aspect comme une catégorie universelle présente dans toutes les langues mais exprimée de façon différente. L'auteur propose un modèle de cinq types d'aspectualité en regroupant toutes les oppositions aspectuelles pouvant qualifier le procès. Il y intègre tous les moyens d'expression de l'aspect - morphologiques, lexicales, même les périphrases verbales. En s'appuyant sur cette approche, nous allons nous focaliser sur la présentation des aspects grammatical et lexical en français et en serbe ce qui nous permettra de rendre compte de la différence de l'aspect dans ces deux langues. Qui plus est, nous mettrons en évidence également le fonctionnement aspectuel du conditionnel et du potentiel - deux formes verbales représentants des (semi-) équivalents dont l'information aspectuelle est très pertinente mais elle n'a pas été assez examinée dans la littérature, notamment dans le cas du potentiel.

\section{SUR L'ASPECT EN FRANÇAIS}

La notion d'aspect représente un sujet problématique dans la littérature française étant donné les nombreuses divergences d'analyse et de terminologie parmi les linguistes. On voit perdurer également le désaccord sur les relations entre les aspects grammatical et lexical, sur les rapports de l'aspect et du temps, sur l'importance de l'aspect dans le système verbal. Toutes les divergences relèvent en fait de l'approche sémasiologique appliquée traditionnellement, même aujourd'hui (Tournadre 2004 :

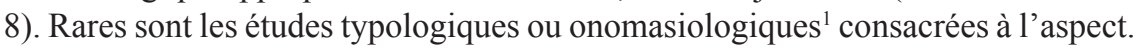
Tournadre (2004) choisit cette approche en traitant de l'aspect comme d'une catégorie universelle présente dans toutes les langues mais exprimée de façon différente. Il accorde une attention particulière à l'interaction et à l'imbrication existant entre les catégories du mode, du temps et de l'aspect en expliquant la liaison entre elles par le fait qu'une même forme verbale porte une valeur aspectuelle dans un contexte mais marque une valeur modale ou temporelle dans un autre contexte. Il propose un modèle de cinq types d'aspectualité en regroupant toutes les oppositions aspectuelles pouvant qualifier le procès. Il y intègre tous les moyens d'expression de l'aspect - morphologiques, lexicales, même les périphrases verbales. Nous soutenons ce type d'approche sauf que nous tenons à distinguer l'aspect grammatical (lié au français) et l'aspect lexical (en vigueur en serbe).

${ }^{1}$ Comrie 1976, Cohen 1989, Dahl 1995. 


\subsection{Sur l'aspect grammatical en français}

Le modèle de l'aspect slave, se fondant sur l'opposition morphologique perfectif / imperfectif (dont il sera question infra) a servi de base pour la théorie des aspects et a été étendu au français et aux autres langues aussi. Cependant, Cohen (1989) et Ducrot (1995) remarquent que ce modèle n'est pas généralisable. Ce fait explique la difficulté (voire le refus) d'introduire la catégorie d'aspect en grammaire française. C'est Guillaume (1929) et des linguistes guillaumiens (P. Imbs, R. Martin, B. Pottier, M. Wilmet, etc.) qui ont accordé une position importante à l'aspect en français (Riegel et al. 1994 : 292). Selon G. Guillaume, l'aspect est de première importance dans la classification verbale, i.e. les catégories aspectuelles précèdent les catégories temporelles : «c'est toujours après avoir assigné au verbe un aspect qu'on lui assigne un temps » (Guillaume $1929: 192)$.

L'auteur élabore sa théorie de l'aspect à partir des notions de tension et d'incidence. Sa définition de l'aspect s'appuie sur la notion de temps impliqué. Guillaume (1929 : 46) d'abord, décrit le verbe comme « un sémantème qui implique et explique le temps ». Le temps impliqué est celui qui est " inhérent au verbe », « intérieur à l'image verbale de langue », tandis que le temps expliqué est celui que le verbe n'emporte pas avec soi par définition, que le discours lui attribue sous la forme du passé, du présent et du futur. Cette distinction du temps impliqué et du temps expliqué correspond à la distinction de l'aspect et du temps (Guillaume, idem.).

La notion de tension est fondamentale dans la conception de l'aspect de G. Guillaume qui entre dans la composition de l'image verbale. L'image verbale est présentée sur la ligne entre deux limites $\mathrm{A}$ et $\mathrm{B}$, dont la tension commence en $\mathrm{A}$ et s'achève en B (Guillaume 1929 : 16) :

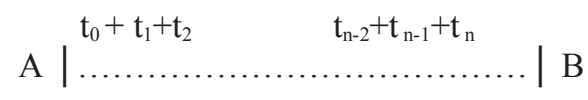

Dans la position initiale $-t_{0}-$ le verbe n'a pas encore dépensé sa tension (en français cette représentation du verbe est exprimée par l'infinitif) $; t_{1}+t_{2 \ldots \ldots .} \quad t_{n-2}+t_{n-1}$ représentent les positions intermédiaires où le verbe a dépensé une partie de sa tension qui correspond à la détension (représentation verbale exprimée par le participe présent) ; et dans la position finale $-\mathrm{t}_{\mathrm{n}}-$ la tension est dépensée jusqu'au bout, il ne reste que la détension qui entre seule dans la composition de l'image verbale (représentation verbale exprimée par le participe passé).

Ainsi Guillaume (1929 : 20) distingue-t-il trois types d'aspect :

1. aspect tensif (simple) qui « représente le verbe en tension »;

2. aspect extensif(composé) qui « sert à renouveler la tension du verbe, au moment où elle expire et à la prolonger au-delà d'elle-même, en extension »; 
3. aspect bi-extensif(surcomposé) qui « reprend en tension, au moment où elle expire, l'extension précédemment obtenue par le même moyen ».

Cette tripartite distinction aspectuelle correspond à l'opposition entre formes verbales simples, composés et surcomposés. Nous ne nous intéresserons ici qu'aux temps de l'indicatif.

$\begin{array}{lll}\begin{array}{l}\text { Forme simple } \\ \text { tension }\end{array} & \begin{array}{l}\text { Forme composée } \\ \text { extension }\end{array} & \begin{array}{l}\text { Forme surcomposée } \\ \text { bi-extension }\end{array} \\ \text { il chante } & \text { il a chanté } & \text { il a eu chanté } \\ \text { il chanta } & \text { il eut chanté } & \text { il eut eu chanté } \\ \text { il chantait } & \text { il avait chanté } & \text { il avait eu chanté } \\ \text { il chantera } & \text { il aura chanté } & \text { il aura eu chanté } \\ \text { il chanterait } & \text { il aurait chanté } & \text { il aurait eu chanté }\end{array}$

Nous ne prendrons pas en compte les formes surcomposées parce qu'elles sont peu employées et peu pertinentes pour notre travail.

Les temps verbaux simples relevant de l'aspect tensif qui «éveille dans l'esprit l'image même du verbe dans son déroulement » (Guillaume 1929 : 21) présentent le procès en accomplissement tandis que les temps verbaux composés, relevant de l'aspect extensif qui " éveille dans l'esprit le déroulement d'une « séquelle » de cette image verbale » (idem.), donnent à voir le procès accompli.

La deuxième distinction aspectuelle en français s'explique à partir de la notion d'incidence.

G. Guillaume classe le passé simple et le futur simple dans les temps incidents alors que l'imparfait et le conditionnel sont classifiés dans les temps décadents. Qu'est-ce que cela signifie ? Le premier groupe des formes verbales donne à voir le procès globalement à partir de sa borne initiale en direction de sa borne finale tandis que le second groupe donne une vision sécante du procès. Distinguons que G. Guillaume considère le conditionnel comme le temps symétrique de l'imparfait et lui réserve la même représentation du procès - sécante.

En somme, on a exposé la problématique de l'aspect grammatical dont l'information aspectuelle est encodée par des morphèmes grammaticaux. En français l'aspect grammatical est exprimé par l'opposition entre les formes simples et composées et par la flexion verbale, notamment par l'opposition ${ }^{2}$ entre l'imparfait et le passé simple.

La théorie guillaumienne sur l'aspect a influencé de nombreux linguistes (entre autres : M. Wilmet, L. Gosselin, J. Bres, etc.). Nous prendrons en compte le travail de Bres (2006) du fait que l'auteur effectue l'analyse concernant l'instruction aspectuelle du conditionnel.

${ }^{2}$ Selon G. Guillaume, l'opposition de ces deux formes verbales est d'ordre temporel, absolument pas d'ordre aspectuel. 
J. Bres aborde son analyse de l'aspect verbal en français en s'appuyant sur les notions de tension, d'extension et d'incidence de Guillaume (1929). Selon l'auteur (2006: 52), les temps verbaux français, sauf les temps prospectifs, peuvent encoder deux types d'instructions aspectuelles :

(i) [+ tension] / [+ extension] qui est marquée morphologiquement par la différence entre formes simples et formes composées, i.e. les formes simples représentent le temps interne au procès en tension, entre les bornes initiale et terminale, et les formes composées représentent le temps interne au procès en extension, à partir de la borne terminale atteinte.

Forme simple

elle lit

il lira

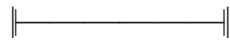

Forme composée

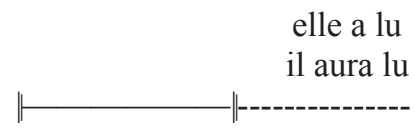

(ii) [+ incidence] / [- incidence] qui « concerne principalement le mode d'inscription du temps interne impliqué par le verbe sur l'imaginaire ligne du temps que construit le discours » (Bres 2006 : 17). Cette instruction aspectuelle ne se réfère qu'à l'époque passée dans les oppositions entre le passé simple (PS) et l'imparfait (IMP) ou entre le passé antérieur (PA) et le plus-que-parfait (PqP). Alors, les deux formes verbales, à savoir le PS et l'IMP, représentent les formes simples relevant de l'aspect tensif, mais donnent à voir le procès différemment. Cela est en vigueur avec les formes composées - le PA et le PqP qui relèvent de l'aspect extensif. En effet, l'IMP et le $\mathrm{PqP}$ représentent le procès de façon sécante (cursive ou dans son cours), c'est-à-dire sans prendre en compte les bornes, tandis que le PS et le PA produisent une vision globale du procès, i.e. le présente de la borne initiale à la borne finale.

Soulignons ici que par rapport à G. Guillaume, J. Bres n'applique la distinction global / sécant qu'aux quatre temps du passé (PS, PA, IMP, PqP).

En examinant la question de l'aspect relevant de la catégorie de l'incidence du conditionnel, Bres (2006) affirme que cette forme « donne une représentation aspectuelle ni globale ni cursive : il est neutre [ \pm incidence] vis-à-vis de cette opposition ». Soulignons que nous partageons la position de J. Bres. Son hypothèse peut être vérifiée par un test de remplacement des formes au passé simple et à l'imparfait par un conditionnel présent (forme verbale simple comme les formes des PS et IMP) :

a. Il était [+ incidence] depuis trois mois à Lille quand il reçut [- incidence], un matin, la visite d'une jeune femme, la sœur de son ancienne maîtresse. (Maupassant, Une passion)

b. Il serait depuis trois mois à Lille quand il recevrait, un matin, la visite d'une jeune femme. 
Ce test démontre que le conditionnel présent peut remplacer à la fois l'imparfait, qui donne à voir le procès de façon sécante et le passé simple, qui en donne une vision globale, ce qui justifie la neutralité aspectuelle du conditionnel présent. Le conditionnel passé comme la forme composée relève de l'aspect extensif, mais lui ${ }^{3}$ aussi est neutre vis-à-vis de la catégorie de l'incidence.

\subsection{Sur l'aspect lexical en français}

L'aspect peut être encodé lexicalement, i.e. il peut relever du sémantisme du lexème verbal. Dans ce cas on parle de l'aspect lexical (ou modalité d'action, mode d'action, Aktionsart chez les linguistes allemands, etc.) s'appuyant sur l'opposition perfectif / imperfectif. En français ce type d'aspect n'est pas systématique, i.e. le verbe peut changer de valeur aspectuelle en fonction du contexte (lire / lire le livre) ou des temps verbaux dans lesquels il est employé. Cependant, hors contexte, on peut distinguer des verbes perfectifs (boire, sortir, gagner) et imperfectifs (aimer, vieillir, chercher) simplement selon leur sens ou selon le procès d'affixation direredire, pleuvoir-pleuviner. En fait, l'aspect lexical en français « correspond au type de procès (activité, état, accomplissement...) exprimé par le lexème verbal et son environnement actanciel » (Gosselin 1996 : 10). L'expression de cet aspect envisage, donc, de prendre en compte les arguments du verbe et d'autres compléments (les auxiliaires, les adverbes entrant en combinaison aspectuelle avec le verbe).

En suivant la classification aspectuelle des verbes proposée par Vendler (1957) on distingue quatre types de procès selon les traits suivants : la stativité, la durée et la télicité. Les signes + ou - se déterminent sur la base des tests syntaxiques de Vendler.

\begin{tabular}{|c|c|c|c|}
\hline & stativité & durée & télicité \\
\hline états & + & + & - \\
\hline activités & - & + & - \\
\hline accomplissements & - & - & + \\
\hline achèvements & - & + & + \\
\hline
\end{tabular}

Nous allons modifier un peu ces tests du fait qu'il y a dans la littérature serbe des tentatives d'application de la classification vendlérienne aux verbes serbes.

Le test de compatibilité avec les adverbes vite et délibérément détermine le trait $[ \pm$ stativité]. Le trait [+ stativité] est attribué aux états (E) tandis que le trait

${ }^{3}$ Bres (2009 : 17) considère également les formes du présent et du futur comme neutres vis-à-vis de la catégorie de l'incidence. 
[- stativité] est accordé aux situations dynamiques des activités (ACT), accomplissements $(\mathrm{ACC})$ et achèvements $(\mathrm{ACH})$ qui entrent sans problèmes en combinaison avec ces adverbes.

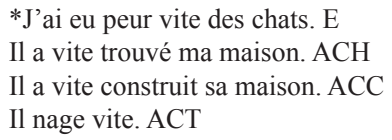

Le test en / pendant indique la télicité du procès. La notion de télicité ${ }^{4}$ est connue depuis Aristote et en linguistique devient répandue surtout grâce aux travaux de Z. Vendler. Un procès est télique (a) s'il est mené vers une limite, un point final qui reste potentiel, alors qu'il est atélique (b) s'il est présenté comme non-borné :

a. Jean s'est promené jusqu'au château.

b. Jean s'est promené dans la forêt.

Le procès télique [+ télicité] entre en combinaison avec en $x$ temps, alors que le procès atélique [- télicité] se combine avec pendant $x$ temps. Le trait [+ télicité] est attribué aux situations dynamiques des $\mathrm{ACC}$ et $\mathrm{ACH}$, alors que le trait [- télicité] est accordé aux E et ACT. semaine. E

Elle a été éperdue de joie pendant une semaine. * Elle a été éperdue de joie en une

J'ai couru pendant une demi-heure. * J'ai couru en une demi-heure. ACT

Nous sommes sortis en deux minutes. ${ }^{* 5}$ Nous sommes sorties pendant deux minutes. ACH

J'ai planté un arbre en une heure. * J'ai planté un arbre pendant une heure. ACC

Le test de compatibilité avec la forme progressive être en train de + infinitif détermine la différence entre certains procès téliques et atéliques. Ainsi les verbes d'ACT et d'ACC sont ceux qui peuvent être employés avec la forme progressive, à la différence des $\mathrm{ACH}$ et des $\mathrm{E}$.

Ils étaient en train de parler. ACT

J'étais en train de traverser le pont. ACC

*J'étais en train de perdre mes clés. ACH

*Elle était en train d'être frileuse. E

Le test de compatibilité avec la forme passer $x$ temps $\grave{a}+$ infinitif indique le trait $[ \pm$ durée]. Le trait [ + durée] est attribué aux situations dynamiques des ACC et $\mathrm{ACT}$, alors que le trait [- durée] est accordé aux E et ACH.

Nous avons passé quelques minutes à marcher. ACT

Elles ont passé deux heures à faire le gâteau. ACC

*Tu as passé dix minutes à sortir de la voiture. $\mathrm{ACH}$

*Le verre a passé une heure à être plein. E

${ }^{4}$ En grec $\tau \dot{\varepsilon} \lambda o \varsigma$ ('la fin').

${ }^{5}$ La phrase en question est tout à fait possible en français, mais avec sens différente. 
Nous n'entrerons pas dans les discussions suscitées par des linguistes (Vetters 1996, GOSSELIN 1996, Recanati et Recanati 1999) concernant la classification vendlérienne étant donné que cela nous éloigne quelque peu du centre de notre analyse - la relation entre l'aspect et le conditionnel.

Abouda (1997) accorde une place importante à l'aspect lexical (au trait $[ \pm$ télique] du verbe) dans son analyse du conditionnel français. L'auteur, d'abord, décrit la différence entre le conditionnel présent (forme simple) et le conditionnel passé (forme composé) comme aspectuelle ${ }^{6}$, et plus précisément comme « une opposition entre l'accompli et le non-accompli» (Abouda 1997 : 406). Le conditionnel présent représente le procès comme non-achevé, i.e. il est «non marqué aspectuellement » (ibid., p. 386), tandis que le conditionnel passé dénote un aspect accompli, c'est-àdire il présente le procès comme achevé. Est-ce que l'aspect lexical (verbes télique vs atélique) joue un rôle dans la détermination de l'aspect grammatical des deux conditionnels ? L. Abouda répond à cette question et démontre qu'il est possible de «prédire directement, dans un certain nombre de cas [dans les complétives], le type de conditionnel dont il s'agit dans un énoncé concret » (ibid., p. 413) moyennant l'aspect lexical (télique $v s$ atélique) et l'aspect grammatical (conditionnel présent vs conditionnel passé).

Par exemple, dans le cas suivant, le verbe au conditionnel passé, se trouvant dans l'emploi temporel, est un verbe télique :

1. Pierre m'avait dit qu'il aurait terminé son travail avant le 30 juin.

Cependant, L. Abouda met en question le type du conditionnel dans le cas où le verbe au conditionnel passé est un verbe atélique :

2. Pierre m'avait dit que Paul aurait été malade.

L'auteur (ibid., p. 407) conclut que « lorsque le verbe est atélique, la forme composée du conditionnel ne peut pas avoir une valeur temporelle »; les seules interprétations possibles y sont les interprétations modales du conditionnel (le conditionnel épistémique, hypothétique, etc.).

\section{SUR L'ASPECT EN SERBE}

Dans la communauté linguistique slave l'aspect joue un rôle beaucoup plus important que dans la communauté linguistique française. En fait, la catégorie grammaticale de l'aspect est attribuée aux langues slaves comme l'expression prototypique où l'aspect du verbe est marqué morphologiquement. Les linguistes serbes (Belić 1925/26, Grubor 1953, Vuković 1967, Maretić 1931, Grickat 1958, Stevanović 1967, Riđanović 1976, Stevanović 1969, Mrazović et Vukadinović

\footnotetext{
${ }^{6}$ Par contre, R. Martin et P. Haillet affirment que cette distinction est d'ordre temporel.
} 
1990, Stanojčić et Popović 1994, Piper et al. 2005) affirment se fait en soulignant que l'aspect représente une caractéristique morphologique inhérente au verbe serbe. Toute forme verbale est associée à l'un des aspects - perfectif ou imperfectif :

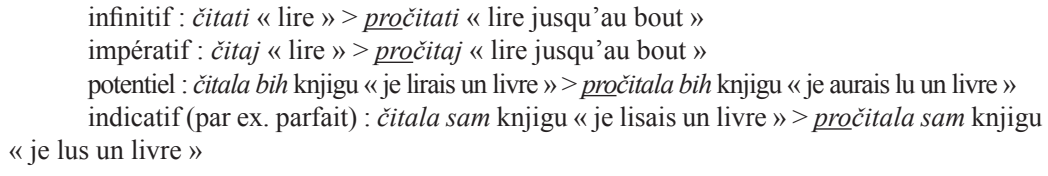

Cette opposition ${ }^{7}$ est constitutive de la catégorie grammaticale de l'aspect sans pouvoir être dissociée du lexique, car elle forme avec celui-ci un ensemble (lexicogrammatical) et elle est indépendante de son expression temporelle (Novakov 2005 : 12). En effet, pour déterminer l'aspect d'un lexème verbal il n'est pas nécessaire de le réaliser dans un temps verbal, car c'est déjà l'infinitif qui est doté du sens aspectuel (imperfectif ou perfectif).

Cependant, certaines restrictions existent, i.e. il est des verbes d'une instruction aspectuelle qui ne peuvent pas participer dans la formation de certaines formes verbales. Par exemple, pour former le participe présent on utilise uniquement des verbes imperfectifs, tandis que le participe passé et l'aoriste sont construits principalement avec les verbes perfectifs. Aussi il existe un nombre restreint de verbes qui selon le contexte peuvent avoir un sens perfectif ou imperfectif (verbes bi-aspectifs).

À l'exception des verbes bi-aspectifs, le verbe serbe se présente sous la forme d'une paire aspectuelle :

čitati « lire » > pročitati « lire jusqu'au bout »
kupiti 《 acheter » > kupovati « être en train d'acheter»

On a souligné les désinences des processus morphologiques (dérivationnels), à savoir la préfixation et la suffixation, qui participent dans la production des couples aspectuels des verbes en serbe. Moyennant ces deux processus un verbe obtient son corrélat d'aspect opposé. Plus précisément, si le verbe est imperfectif, son corrélat perfectif est dérivé soit par préfixation :

trčati « courir »> dotrčati « accourir »; plivati « nager » > preplivati « traverser à la nage », soit par suffixation : kucati « taper » > kucnuti « taper une fois ».

Au cas où le verbe est perfectif, son corrélat imperfectif s'obtient uniquement par suffixation : dati « donner » > davati « être en train de donner » / « donner régulièrement »,

kupiti « acheter » > kupovati « être en train d'acheter » / 《 acheter régulièrement ».

Il est à noter que les préfixes représentent les marqueurs de la perfectivité (Stevanović 1969, Tanasić 2005). Ce type d'affixes est en nombre limité étant

${ }^{7}$ « On peut bien considérer le système aspectuel slave comme organisé à partir de l'opposition perfectif-imperfectif » (Novakova $2001: 12$ ). 
donné qu'ils appartiennent à une classe fermée ${ }^{8}$. D'autre part, les suffixes marquent l'imperfectivité (-va- dans davati, -ja- dans dobijati «être en train d'obtenir », $-a$ - dans padati « être en train de tomber », etc.). L'unique exception représente le suffixe semelfactif - $n u$ - qui produit des verbes perfectifs (-nu- dans kucnuti « taper une fois »).

S'agissant de la base à laquelle se rattachent les affixes mentionnés, elle peut être perfective ou imperfective, simple ou dérivée. Les suffixes imperfectifs se rattachent généralement à des bases perfectives pour former des verbes imperfectifs, alors que les préfixes peuvent sélectionner des bases aussi bien imperfectives que perfectives afin de dériver les verbes perfectifs (Ašić et Stanojević $2011: 8$ ). Par exemple, le préfixe pri- se rattache le plus souvent à des bases imperfectives (pisati « écrire » > pripisati « attribuer »), mais il peut aussi être attribué à des bases perfectives (dodati « rajouter » > pridodati « rajouter en supplément »).

Cet exemple révèle aussi une autre fonction des préfixes, i.e. à part de la perfectivisation, les préfixes peuvent changer le sens lexical du verbe dérivé : pisati « écrire » > napisati « écrire qqch jusqu'au bout »; pripisati « attribuer »; prepisati « copier »; propisati « prescrire »; dopisati « rajouter à ce qui a été écrit ». Pour cette raison le statut des préfixes est mis en question dans la littérature (Forsyth 1970, Binnick 1991, Schoorlemmer 1995), la question de savoir s'il existe des préfixes purement aspectuels dans les langues slaves, mais nous n'entrerons pas ici dans ce débat. Par contre, les suffixes aspectuels fonctionnent uniquement comme marqueurs aspectuels, à la différence des préfixes.

Selon un certain nombre de linguistes (Grubor 1953, Riđanović 1976, Stevanović 1979, Novakov 2005) la distinction entre deux aspects des verbes serbes n'inclut pas toutes les nuances aspectuelles dérivées par les verbes serbes. C'est Grubor (1953) qui a remarqué le premier ce fait en distinguant deux classes des verbes serbes, à savoir les verbes évolutifs et les verbes d'état, chacune d'entre elles divisée en verbes imperfectifs et verbes perfectifs. L'auteur obtient ainsi quatre types de verbes, ce qui lui permet de déterminer plus précisément les sens des couples aspectuels des verbes perfectifs et imperfectifs. Les types de verbes qu'indique Grubor sont analogues à la classification aspectuelle entreprise par Vendler en 1957. L'auteur a eu recours, comme Vendler également, à l'ancienne division d'Aristote en procès dynamiques (verbes évolutifs) et procès statiques (verbes d'état). Mais, au fond de cette division verbale proposée par Grubor il y a, en fait, la distinction entre l'aspect comme catégorie grammaticale et le mode de procès (Aktionsart) comme catégorie lexicale. Les études contrastives anglo-serbes de Ridjanović (1976) et Novakov (2005) insistent notamment sur cette distinction et décrivent les verbes

${ }^{8}$ Maretić (1961) et Stevanović (1979) énumèrent seize préfixes tandis que d'autres auteurs indiquent un nombre plus grand, comme par exemple Klajn (2002), vingt-quatre. 
serbes dans le cadre vendlérien. L'application de la classification de Vendler aux verbes serbes est représentée dans le tableau ci-dessous :

\begin{tabular}{|c|c|c|c|}
\hline & stativité & durée & télicité \\
\hline états & + & + & - \\
\hline activités & - & + & - \\
\hline accomplissements & - & + & + \\
\hline achèvements & - & - & + \\
\hline
\end{tabular}

On peut remarquer dans ce tableau que les caractéristiques sémantiques des verbes serbes et français sont analogues :

1. Les traits [+ stativité], [+ durée], [- télicité] sont attribués aux états (E). En serbe, les verbes d'état sont représentés par les verbes imperfectifs :

\footnotetext{
- beleti se, crneti se, znati, voleti, koštati, ljutiti se, sijati, nalaziti se (être blanc, noircir savoir, aimer, coûter, se fâcher, briller, se trouver, etc.).
}

- et le verbe imperfectif être en combinaison avec un adjectif; un adverbe : biti + lep, pametan, zdrav, plav, bolestan, uvređen, nasmejan; daleko, blizu, tu, tamo (être beau, intelligent, sain, blond, malade, offensé, souriant ; loin, proche, ici, là).

2. Les traits [- stativité], [+ durée], [- télicité] sont accordés aux activités (ACT). Les verbes imperfectifs : krečiti, trčati, hodati, tražiti, gledati, čistiti, kijati, lupati, plivati, etc. (peindre, courir, marcher, chercher, regarder, nettoyer, éternuer, frapper, nager, etc.) indiquent ACT.

3. Les accomplissements (ACC) sont qualifiés par les traits [- stativité], [+ durée], [+ télicité]. Dans le groupe des verbes d'ACC on peut classer les verbes suivants : okrečiti sobu, napisati članak, preći ulicu, preplivati reku, počistiti kuću, naučiti nemački, nacrtati dinosaurusa, pretrčati 800 metara, etc. (peindre une chambre (jusqu'au bout), écrire un article (jusqu'au bout), traverser la rue, traverser à la nage la rivière, nettoyer la maison (jusqu'au bout), apprendre allemand (jusqu'au bout), dessiner un dinosaure (jusqu'au bout), courir 800 m (jusqu'au bout), etc.).

4. Les achèvements $(\mathrm{ACH})$ possèdent les traits [- stativité], [- durée], [+ télicité]. Les ACH peuvent être encodés par les verbes suivants : pobledeti, razboleti se, ozdraviti, zabeleti se, saznati, umreti, roditi se, izgubiti (ključeve), naći (ključeve), ući, izaći, stići, pasti, reći, ustati, leći (blêmir, tomber malade, se remettre, commencer à être blanc, apprendre, mourir, naître, perdre (les clés), trouver (les clés), entrer, sortir, arriver, tomber, dire, se lever, se coucher, etc.)

L'objectif de notre présentation des classes verbales aspectuelles en serbe selon le modèle vendlérien est en fait de souligner le rôle des affixes dérivationnels 
dans le domaine de l'aspect en serbe. Même si les tableaux démontrent l'analogie des caractéristiques sémantiques des verbes serbes et français, les verbes serbes exemplaires des $\mathrm{E}, \mathrm{ACT}, \mathrm{ACC}$ et $\mathrm{ACH}$ exposés ci-dessus révèlent une importante différence manifestée par ces classes en serbe et en français - l'importance de la dérivation verbale dans la langue serbe. Les affixes dérivationnels sont beaucoup plus présents en serbe qu'en français, voire omniprésents. Les préfixes apparaissent dans toutes les classes et notamment dans les ACC et les ACH ; les suffixes imperfectifs (surtout $-v a-,-a$ - comme les plus fréquents) dans la classe des ACT et les E, et le suffixe perfectif - $n u$ - dans la classe des ACH.

On peut conclure que s'appuyer sur la quadripartite classification aspectuelle n'est pas obligatoire en serbe, étant donné qu'il suffit d'avoir recours au sémantisme du verbe même et aux instructions des affixes dérivationnels.

\subsection{L'aspect et le potentiel}

Nous avons vu que le conditionnel est neutre vis-à-vis de l'opposition globale - cursive. On pourrait dire également que le potentiel se caractérise par la neutralité aspectuelle. Plus précisément, cette forme verbale peut donner les deux types de représentation aspectuelle dans le cadre de la catégorie d'incidence, à savoir les représentations globale et cursive d'un procès. Évidemment, en serbe, le potentiel exprime ces deux visions du procès en entrant en combinaison avec les verbes perfectifs et imperfectifs. Cette caractéristique du potentiel entraîne certaines ambiguités dont il sera question infra ce qui rend le rôle de l'aspect dans le cas du potentiel très pertinent.

Dans la littérature serbe, on rencontre deux formes du potentiel, simple et composée. Le potentiel simple représente la combinaison du passé simple du verbe biti ('être') et du participe passé du verbe conjugué :

ja bih čitao - je fus lu $<$ je lirais

Le potentiel composé ou du passé (Stevanović 1969 : 692) se construit sur le potentiel simple du verbe biti ('être') et le participe passé du verbe conjugué :

ja bih bio čitao - je fus été lu $<$ j'aurais lu

Il ne connait que la valeur modale exprimée dans le passé. Cependant, cette forme du potentiel est disparue dans la langue contemporaine. On n'utilise aujourd'hui que la forme simple du potentiel. Cela s'explique par le fait que le potentiel simple peut être employé dans les contextes où figure le potentiel composé sans aucun changement de sens : 
(1) Može biti da bi bolje bio učinio da je izostavio9. Peut-être il aurait fait mieux s'il l'avait oublié.

(2) Može biti da bi bolje učinio da je izostavio. Peut-être il aurait fait mieux s'il l'avait oublié.

Qui plus est, les grammairiens affirment ce fait en insistant que la forme composée du potentiel n'est pas justifiée pour la raison déjà indiquée supra (Stevanović 1969 : 693). Cependant, cette attitude n'explique pas la nécessité préalable de ladite forme du potentiel dans la langue serbe. Soulignons aussi que le développement de la problématique en question manque dans la littérature.

Nous posons (Dodig, en cours) que l'aspect morphologique en serbe et le procès de grammaticalisation sont les facteurs explicatifs de la disparition de la forme composée du potentiel. Il est à noter que l'aspect grammatical a existé en serbe. La distinction forme simple / forme composée du potentiel, i.e. aspect tensif / extensif, était importante en serbe, ainsi que l'opposition imparfait / passé simple, mais les formes verbales, à savoir le potentiel composé et l'imparfait, sont tombées en désuétude. En fait, la langue serbe a grammaticalisé l'aspect grammatical en aspect morphologique. La dominance de l'aspect morphologique a entraîné la disparition de l'opposition forme simple / forme composée du potentiel. Concluons qu'en serbe contemporain ladite distinction n'est pas pertinente. Les recherches diachroniques supplémentaires sont nécessaires pour pouvoir déterminer précisément le chemin d'évolution des aspects en question.

Rappelons que le potentiel comme le conditionnel peut avoir des emplois temporels et des emplois modaux. Selon notre classification (Dodig, en cours), on distingue le potentiel modal, le potentiel habituel ${ }^{10}$ désignant la répétition dans le passé qui représente le cas transitif entre les potentiels modal et temporel, et le potentiel temporel ${ }^{11}$ figurant dans les non-téléonomiques ${ }^{12}$. S'agissant de l'instruction aspectuelle de ces usages du potentiel, ces formes peuvent se combiner soit avec les verbes perfectifs soit avec les verbes imperfectifs :

a. potentiel modal : Pročitala bih knjigu za dva sata. (J'aurais lu le livre.) pročitati $=$ avoir lu

b. potentiel habituel : Čitala bih knjigu dva sata. (Je lirais le livre.) čitati = lire

c. potentiel temporel : Prvo su putevi bili osposobljeni za saobraćaj da $b i$ potom krenula i temeljna rekonstrukcija. (Les routes ont d'abord été

\footnotetext{
${ }^{9}$ Exemple emprunté à V. Karadžić, Laži i opadanja.

${ }^{10}$ Dans la littérature on ne rencontre que l'appelation - potentiel temporel.

${ }^{11}$ Cet emploi du potentiel a été répertorié pour la première fois par Asic et Dodig (2015).

${ }^{12}$ Type particulier des propositions qui ont la forme syntaxique des propositions finales (téléonomiques), mais dont la fonction est différente.
} 
remises en état de service pour qu'ensuite une reconstruction substantielle fût mise en œuvre.) krenuti = être mis en œuvre

d. potentiel temporel : Kosovski Srbi učestvovali su, u izvesnom broju u Prvom srpskom ustanku, da bi kasnije, 1820 - ih, sa centralnog Kosova bili raseljavani surovim merama odmetnutih albanskih paša. (Un certain nombre de Serbes du Kosovo ont participé à la Première insurrection serbe ; plus tard, dans les années 1820, ils seraient déplacés du Kosovo central par les mesures sévères des pachas albanais rebelles.) raseljavati $=$ être en train de déplacer

Nous avons remarqué que l'instruction aspectuelle peut contribuer à distinguer les emplois du potentiel. Consultons les exemples suivants :

(3) Kad bi našao vremena, pročitao bih knjigu za dva sata. (Si j'en trouvais le temps, j'aurais lu ce livre en deux heures.)

(4) Kad bi našao vremena, čitao bih knjigu dva sata. (Quand j'en trouvais le temps, je lisais le livre pendant deux heures.)

Le verbe imperfectif au potentiel dans le cas de 4 indique qu'il s'agit plutôt du potentiel habituel tandis que le verbe perfectif employé dans l'exemple 3 révèle le potentiel modal (hypothétique).

S'agissant seulement du potentiel habituel, nous trouvons dans la littérature les données d'une importance signifiante de l'aspect :

(5) Ponekad bih otišao da vidim pocrnjele zidove i mrtve oči mrtvog zdanja, u kojem nikako nisam mogao da zamislim bivđe stanovnike: kao da je bilo pusto odvajkada. (M. Selimović, Tvrđava)

(5') J'allais quelquefois contempler les murs noircis et les yeux éteints de la maison morte, qui semblait avoir toujours été vide: je ne parvenais pas à imaginer ses habitants, je ne me voyais plus moi-même dans cette maison. (M. Selimović, La Forteresse)

(6) Kad bih našao vremena, iđao bih do Darive ili Kozje ćuprije i, sjedeći na obali, sluđao kako voda teče. (M. Selimović, Tvrđava)

(6') Quand j'en trouvais le temps, j'allais au bord de la Dariva, ou je poussais jusqu'au pont des Chèvres et, assis sur la rive, j'écoutais couler l'eau. (M. Selimović, Tvrđava)

Dans ces exemples le potentiel est utilisé, comme cela a déjà été expliqué dans la littérature (Stevanović 1969 : 691 ; Ivić 2008 : 60 ; Piper et al. 2005 : 461 ; etc.) pour exprimer le caractère habituel d'un procès, i.e. pour marquer la répétition régulière d'une action dans le passé.

Selon Ivić (2008), on utilise en serbe soit le parfait des verbes imperfectifs, soit le potentiel pour marquer la répétition régulière d'une action dans le passé. 
L'emploi de ces deux formes verbales est fondé sur l'opposition : «l'information strictement factuelle de la répétition de l'action - la représentation émotionnelle de l'action ( l'évocation des souvenirs) » (Ivić $2008: 60$ ) où le parfait figure dans le premier cas et le potentiel dans le deuxième. Piper et al. $(2005: 461)$ remarquent que, concernant l'expression du passé non référentiel, le potentiel est la forme la plus appropriée à marquer les actions accomplies. Autrement dit, dans le cas où le prédicat est formé du verbe perfectif, le potentiel représente l'unique forme pour exprimer le passé non référentiel. S'il s'agit du verbe imperfectif, le potentiel partage cette fonction avec le parfait en produisant l'effet stylistique déjà mentionné - l'évocation des souvenirs. Évidemment, le potentiel des verbes imperfectifs ne marque pas obligatoirement la répétition dans le passé avec cet élément sémantique - la représentation émotionnelle de ce passé. Il s'agit de sa capacité à styliser l'action répétée dans le passé. On peut constater que le potentiel habituel des verbes perfectifs ou imperfectifs désigne la valeur itérative d'une action dans le passé en produisant des effets stylistiques à la différence de son concurrent dans cet emploi - le parfait.

Ađić (2007) ajoute une autre particularité du potentiel exprimant ladite valeur concernant l'aspect. En fait, il existe en serbe des verbes perfectifs (par ex. pognuti (il se pencha) qui ne possèdent pas leurs équivalents imperfectifs pour exprimer l'itération. Comment pourraient-ils exprimer alors la répétition dans le passé? Ce n'est pas possible d'atteindre par le parfait perfectif (b). T. Ađić voit la solution dans le potentiel (a) :

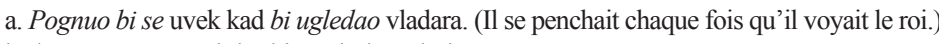

Ensuite, le parfait des verbes imperfectifs ne peut pas exprimer la progression temporelle, à la différence du parfait perfectif. Elle peut être inférée par l'instruction pragmatique, mais ce n'est pas toujours le cas. Ce fait représente un grand problème dans le cadre des propositions temporelles exprimant à la fois la succession (progression temporelle) et la répétition dans le passé.

(7) Čim je ušao u učionicu, đaci su se utišali. Dès qu'il entra ${ }^{13}$ dans la classe, les élèves se sont tus.

(8) ?Čim je ulazio u učionicu, đaci su se utišavali. ?Dès qu'il entrait dans la classe, les élèves se taisaient.

\footnotetext{
${ }^{13}$ Rappelons que dans la langue française la catégorie de l'aspect est encodée dans la plupart des cas par les temps verbaux mêmes, tandis que dans la langue serbe celle-ci est exprimée morphologiquement (moyennant l'opposition des verbes perfectifs - imperfectifs: čitati-pročitati, kupiti-kupovati). Ainsi, utilisons-nous l'opposition entre le passé simple et l'imparfait pour refléter la différence entre le verbe perfectif et imperfectif.
} 
Dans le cas de (7), les prédicats au parfait perfectif sont mis en rapport de la progression temporelle : l'action de la subordonnée introduite par la conjonction dès que se déroule avant celle de la principale. L'exemple (9) est inacceptable parce que le parfait imperfectif donne l'instruction de simultanéité qui est en conflit avec la signification de la conjonction temporelle dès que (antériorité immédiate). Constatons que, dans les propositions temporelles désignant la succession de deux événements, le parfait des verbes perfectifs exprime la progression temporelle tandis qu'elle est exclue au parfait des verbes imperfectifs, mais les deux ne peuvent pas exprimer à la fois la succession et la répétition. On a, donc, besoin d'une forme verbale exprimant la progression temporelle et la répétition dans le passé et en même temps la perfectivité - bref, on aura affaire au potentiel :

(9) Čim bi ušao u učionicu, đaci su se utišavali. Chaque fois qu'il entrait dans la classe, les élèves se taisaient.

Nous ajoutons que, dans la plupart des cas, le potentiel habituel entre le plus facilement en combinaison avec les verbes perfectifs qu'avec les verbes imperfectifs, ce que l'on peut remarquer dans les exemples supra.

En ce qui concerne le potentiel intrinsèquement temporel figurant dans les non-téleonomiques, nous avons remarqué dans notre corpus, constitué de 300 occurrences du potentiel temporel, que ce type de potentiel se combine le plus souvent avec les verbes perfectifs :

(10) Ciceron iz Rima odlazi u Brindizi, a potom boravi sedam meseci u Solunu, da bi potom u jesen stigao/*bi stizao u Drač. (Mostovi : časopis za prevodnu književnost Udruženja književnih prevodilaca Srbije, broj 103)

Cicéron part de Rome à Brindisi, et puis passe un séjour de sept mois à Thessalonique, pour à l'automne, arriver /*être arrivé à Durres.

(11)Japan je, pošto se predao, okupiran od strane američkih snaga, da bi potom postao/*bi postajao jedan od najvažnijih američkih saveznika. (Politika, 07.08.2008.)

Après la reddition, le Japon a été occupé par les forces américaines, pour devenir /*avoir devenu ensuite l'un des plus importants alliés américains.

Il est possible d'employer le potentiel des verbes imperfectifs dans les nontéléonomiques :

(12)Kosovski Srbi učestvovali su, u izvesnom broju u Prvom srpskom ustanku, da $b i$ kasnije, 1820 - ih, sa centralnog Kosova bili raseljavani surovim merama odmetnutih albanskih paša. (Udžbenik istorije za VII razred Osnovne škole) 
Un certain nombre de Serbes du Kosovo ont participé à la Première insurrection serbe ; plus tard, dans les années 1820, ils seraient déplacés du Kosovo central par les mesures sévères des pachas albanais rebelles.

Cependant, on ne rencontre que de 3\% de ces cas dans notre corpus et la plupart des exemples se trouvent en forme passive.

Pourquoi le potentiel dans les non-téléonomiques est-il le plus souvent en combinaison avec les verbes perfectifs ? À notre avis, c'est pour la raison de la relation finale qui existe toujours entre les deux événements se succédant $\left(\mathrm{e}_{1}\right.$ et $\left.\mathrm{e}_{2}\right)$, malgré le fait qu' $e_{2}$ introduit par le potentiel figurant dans les non-téléonomiques, ne représente pas un but motivant l'existence d' $\mathrm{e}_{1}$.

La dernière observation qu'on aimerait faire à propos de l'aspect et le potentiel temporel est liée au phénomène de la négation. La non-téléonomique serbe refuse la négation dans la majorité des cas à la différence du conditionnel français dans son emploi non-téléonomique :

(13) Il [Christophe Moreau] finit trente-deuxième, mais une fois encore premier Français de l'étape. Le massif du Sancy était - déjà - un peu trop haut pour nos grimpeurs. Le triomphe à Super-Besse, 1289 mètres d'altitude, ne serait pas pour eux. (Le Monde, 12 juillet 2008, racontant l'étape du 11 juillet)

On (Kristof Moro) završio je kao trideset drugi, ali ipak kao prvi Francuz. Masif Sansi je bio malo previsok za naše planinare. Trijumf na Super-Besu, 1289m nadmorske visine, nije za njih.

Dans la traduction de l'exemple (13) on n'a pas opté pour la non-téléonomique mais pour une phrase juxtaposée au présent narratif.

Il semble que la négation dans les non-téléonomiques est possible uniquement avec les verbes d'achèvement dénotant la progression temporelle (ce phénomène est connu dans la littérature comme la négation du rupture ${ }^{14}$, de Saussure 1998 ; Ašić 2011) :

(14)Okrenula se ka njemu $\quad$ da mu potom ipak ne bi uputila pozdrav.

Elle se tourna vers lui ; pourtant ensuite elle ne lui envoya pas le salut / sans lui envoyer ensuite le salut.

\footnotetext{
${ }^{14}$ Dans la négation du rupture on assigne une référence temporelle au prédicat sous la portée de la négation et, par conséquent le temps avance : Jacques emprunta le boulevard périphérique; ensuite, il ne s'arrêta pas à la station-service. (DE SAUSSURE 1998)
} 


\section{BILAN}

Pour résumer, en français l'aspect grammatical est encodé soit par l'opposition entre les formes simples et composées (référant à la catégorie de la tension), soit par la flexion verbale (référant à la catégorie de l'incidence) par lesquelles s'exprime l'opposition perfectif / imperfectif moyennant notamment l'imparfait et le passé simple, alors qu'en serbe c'est l'aspect lexico-morphologique inhérent au verbe serbe qui exprime l'opposition perfectif / imperfectif à l'aide des paires aspectuelles des verbes imperfectifs et perfectifs. D'un côté il y a des verbes imperfectifs et perfectifs simples, non dérivés et, de l'autre côté, les processus dérivationnels, à savoir la préfixation et la suffixation, produisant leurs corrélats aspectuels dérivés. L'aspect lexical mentionné supra existe également en français, mais ce type d'aspect n'est pas systématique du fait que le verbe peut changer de valeur aspectuelle en fonction du contexte (lire / lire le livre) ou des temps verbaux dans lesquels il est employé. En fait, l'aspect lexical en français correspond au type de procès - activités, états, accomplissements et achèvements.

S'agissant du conditionnel, on peut conclure que cette forme donne une représentation aspectuelle neutre, i.e. il peut représenter le procès aussi bien perfectivement qu'imperfectivement. Étant donné que le potentiel peut être employé avec les verbes des deux instructions aspectuelles (perfective et imperfective) la même conclusion d'aspectualité neutre s'impose également pour cette forme verbale.

Nous avons également démontré le rôle de l'aspect comme un critère distinctif permettant de déterminer le type des emplois du potentiel.

Nous présentons notre résumé dans les tableaux ci-dessous : $:^{15}$

\begin{tabular}{|c|c|c|c|}
\hline & $\begin{array}{c}\text { Aspect } \\
\text { grammatical }\end{array}$ & $\begin{array}{c}\text { Aspect lexical } \\
\text { (sémantique, } \\
\text { préfixal, infixal) }\end{array}$ & $\begin{array}{c}\text { Aspect lexical } \\
\text { (Aktionsart) }\end{array}$ \\
\hline français & + & + & + \\
\hline serbe & $-{ }^{15}$ & + & + \\
\hline
\end{tabular}

${ }^{15} \mathrm{Ce}$ type d'aspect a été exprimé en serbe par l'opposition imparfait / aoriste. Cependant, l'imparfait est tombé en désuétude. 


\begin{tabular}{|c|c|c|c|c|}
\hline & \multicolumn{2}{|c|}{$\begin{array}{c}\text { Aspect } \\
\text { grammatical }\end{array}$} & \multirow{2}{*}{$\begin{array}{c}\text { Aspect lexical } \\
\text { (sémantique, } \\
\text { préfixal, infixal) }\end{array}$} & $\begin{array}{c}\text { Aspect lexical } \\
\text { (Aktionsart) }\end{array}$ \\
\cline { 2 - 3 } & tension & incidence & - & $+( \pm$ télicité $)$ \\
\hline conditionnel présent & + & \pm & - & $+( \pm$ télicité $)$ \\
\hline conditionnel passé & - & \pm & \pm & - \\
\hline potentiel & \multicolumn{2}{|c|}{-} & - & - \\
\hline
\end{tabular}

\section{BIBLIOGRAPHIE}

Abouda 1997 : Recherches sur la syntaxe et la sémantique du conditionnel en français moderne, thèse de doctorat, Paris VII.

Ađić 2007 : „S one strane potencijala - novi pristupi objašnjenju upotrebe potencijala za označavanje ponavljanja u prošlosti”, Južnoslovenski filolog $36,1,137-150$.

Ašić et Stanojević 2006 : Stanojević Veran i Ašić Tijana, Semantika i pragmatika glagolskih vremena u francuskom jeziku, Kragujevac : Filološkoumetnički fakultet.

Ašić et Stanojević 2011 : T. Ašić i V. Stanojević, „,Vremenske upotrebe kondicionala u francuskom i srpskom jeziku", Srpski jezik, književnost, umetnost, Kragujevac : FILUM, 367-379.

Belić 1925/26 : A. Belić, „Aorist imperfektivnih glagola”, Južnoslovenski filolog $V$, Beograd, 170-182.

Binnick 1991 : R. Binnick, Time and the Verb. A Guide to Tense and Aspect, New York - Oxford, Oxford University Press.

Bres 2006 : J. Bres, Les temps de l'indicatif, Paris : Ophrys.

Cohen 1989 : D. Cohen, L'aspect verbal, Paris, Presses universitaires parisiennes.

Dahl 1985 : Ö. Dahl, Tense and Aspect Systems, Basil Blackwell, Oxford, New York.

Genette 1972 : G. Genette, Figures III, Paris : Seuil.

Gosselin 1996 : L. Gosselin, Sémantique de la temporalité en français, un modèle calculatoire et cognitif du temps et de l'aspect, Paris : Duculot.

Grickat 1958 : I. Grickat, „O nekim vidskim osobenostima srpskohrvatskog glagola", Južnoslovenski filolog XXII, 65-130.

Grubor 1953 : Đ. Grubor, Aspektna značenja, Zagreb : JAZU. 
Guillaume 1929 : G. Guillaume, Temps et verbe : théorie des aspects, des modes et des temps, Paris, H. Champion.

Ivić 2008 : M. Ivić, Lingvistički ogledi, Beograd : Biblioteka XX vek.

Maretić 1931 : T. Čaretić, Gramatika i stilistika hrvatskoga ili srpskoga književnog jezika, Zagreb : Matica hrvatska.

Mrazović et Vukadinović 1990 : P. Mrazović i Z. Vukadinović, Gramatika srpskohrvatskog jezika za strance, Novi Sad : Dobra vest.

Novakov 2005 : P. Novakov, Glagolski vid i tip glagolske situacije u engleskom $i$ srpskom jeziku, Novi Sad : Futura publikacije.

Lukajić 2014 : D. Lukajić, L'aspect perfectif et la télicité : une comparaison entre les classes verbales en français et en serbe, http://etudesslaves.edel. univ-poitiers.fr/index.php?id=730, 1.1.2015.

Piper et al. 2005 : P. Piper i dr., Sintaksa savremenog srpskog jezika : prosta rečenica, u redakciji Milke Ivić, Beograd : Institut za srpski jezik SANU, Beogradska knjiga - Novi Sad : Matica srpska.

Riegel et al. 1994 : Riegel Martin et al., Grammaire méthodique de français, Paris, Quadrige - Presses Universitaires de France.

Riđanović 1976 : M. Riđanović, A Synchronic Study of Verbal Aspect in English and Serbo-Croatian, Cambridge, Massachuset : Slavica.

Sperber et Wilson 1986: D. Sperber et D. Wilson, Relevance: Communication and cognition, Oxford: Blackwell; Cambridge, Mass.: Harvard University Press.

Stanojčić et Popović 1994 : Ž. Stanojčić i Lj. Popović, Gramatika srpskog jezika, Beograd: Zavod za udžbenike i nastavna sredstva.

Stevanović 1967 : M. Stevanović, Funkcije i značenja glagolskih vremena, Posebna izdanja Srpske akademije nauka i umetnosti, Odeljenje literature i jezika, knj. 20, Beograd: SANU.

Stevanović 1969 : M. Stevanović, Savremeni srpskohrvatski jezik II, Sintaksa, Beograd: Naučna knjiga.

Tournadre $2004:$ N. Tournadre, Typologie des aspects verbaux en intégration à une théorie du TAM, Bulletin de la Société linguistique de Paris, $X C I X, 7-68$.

Vendler 1957 : Vendler Zeno, "Verbs and Times", The Philosophical Review 66 : 143-160, reprinted in: Vendler Zeno (1967): Linguistics in Philosophy, Ithaca, New York: Cornell University Press, 97-121.

Vuković 1967 : J. Vuković, Sintaksa glagola, Sarajevo : Zavod za izdavanje udžbenika. 


\section{О АСПЕКТУ У ФРАНЦУКОМ И СРПСКОМ ЈЕЗИКУ И АСПЕКТУАЛНОЈ ИНСТРУКЦИЈИ КОНДИЦИОНАЛА И ПОТЕНЦИЈАЛА}

\section{Резиме}

Време, начин и аспект представљају уско повезане категорије неопходне за опис једног глаголског облика. Ми ћемо се у овом раду бавити питањем аспекта, и то у српском и француском језику. Наш циљ је да покушамо да уочимо и објаснимо разлике које се тичу наведене категорије у овим језицима, што ће нам омогућити да сагледамо и аспектуалне инструкције глаголских облика - кондиционала у француском и потенцијала у српском језику.

Кључне речи: граматички аспект, лексички аспект, француски кондиционал, српски потенцијал.

Милана Додиг 